\title{
Hadronic molecules with hidden charm and bottom
}

\author{
Feng-Kun Guo ${ }^{1, \star}$ \\ ${ }^{1}$ CAS Key Laboratory of Theoretical Physics, Institute of Theoretical Physics, Chinese Academy of Sciences, \\ Beijing 100190, China
}

\begin{abstract}
Many of the new structures observed since 2003 in experiments in the heavy quarkonium mass region, such as the $X(3872)$ and $Z_{c}(3900)$, are rather close to certain thresholds, and thus can be good candidates of hadronic molecules, which are loose bound systems of hadrons. We will discuss the consequences of heavy quark symmetry for hadronic molecules with heavy quarks. We will also emphasize that the hadronic molecular component of a given structure can be directly probed in long-distance processes, while the short-distance processes are not sensitive to it.
\end{abstract}

\section{Introduction}

Hadronic molecules are analogues of the light nuclei. They can be treated to a good approximation as composed systems made of two or more hadrons which are bound together via the strong interactions. Thus, their size, $\sim 1 / \sqrt{2 \mu E_{B}}$ with $\mu$ and $E_{B}$ the reduced mass of the constituent hadrons and the binding energy, respectively, should be large so that the constituent hadrons can be recognized separately. They are thus located close to the corresponding thresholds of the strongly coupled hadrons. Hadronic molecules are interesting for several reasons:

(1) They are one type of realizations of color-neutral objects and the investigation of how hadronic molecular spectrum is organized would lead to a better understanding of the strong interactions at low energies.

(2) One can get important information for low-energy hadron-hadron interaction.

(3) Since they are close to thresholds, one has a good separation of energy scales $\sqrt{2 \mu E_{B}} \ll \Lambda_{\text {hadron }}$, where $\Lambda_{\text {hadron }}$ is typically a hadronic scale of the order of a few hundreds of $\mathrm{MeV}$, so that they can be studied using techniques of effective field theory (EFT). One can make model-independent statements about the probability of a near-threshold structure to be a bound state of the corresponding hadrons if they are in an $S$-wave.

(4) There have been a lot of new structures observed since 2003 in the heavy quarkonium mass region which do not fit in the conventional quarkonium spectrum, see Fig. 1. They are generally dubbed as $X Y Z$ states. Some of them are close to open-flavor thresholds and thus good candidates of hadronic molecules.

^e-mail: fkguo@itp.ac.cn 


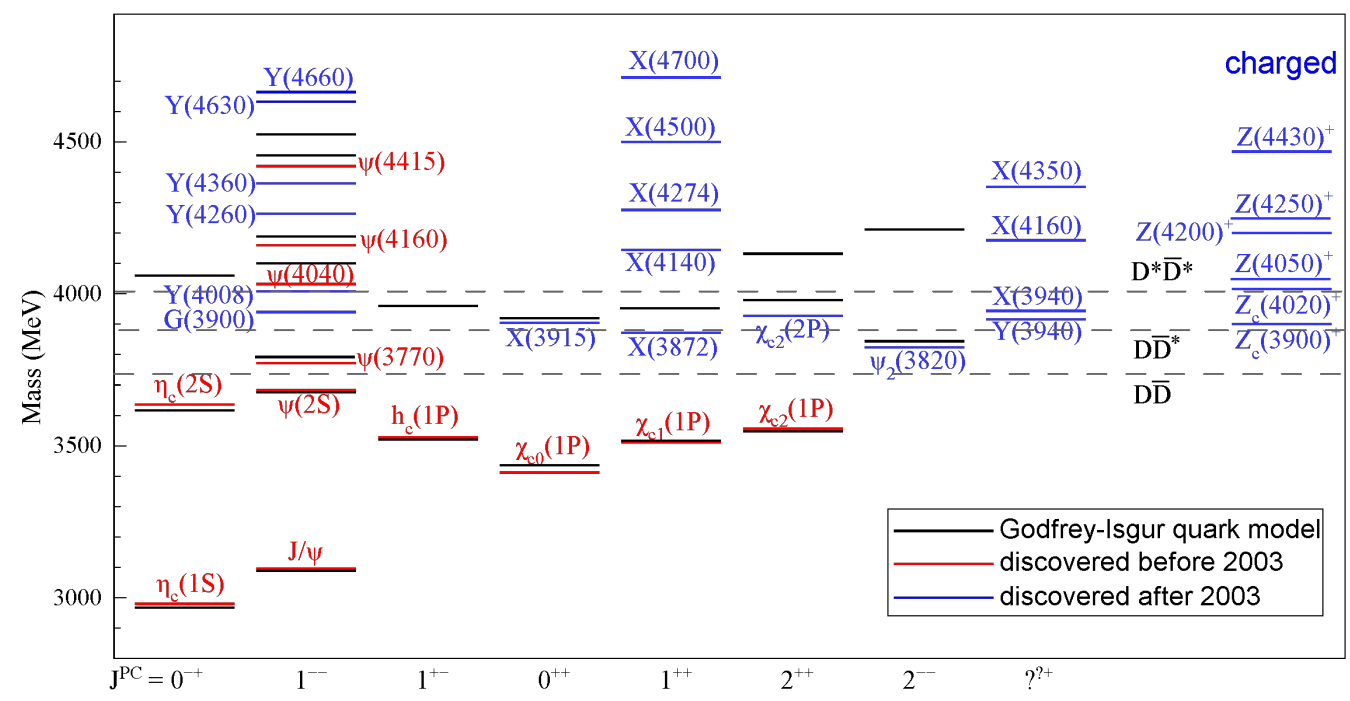

Figure 1. Meson specturm in the charmonium mass region. The black lines are those charmonia predicted in quark model by Godfrey and Isgur [1]. The red lines indicate the states discovered before 2003, and the blue ones correspond to the structures reported after 2003. A few open-charm thresholds are also shown as dashed lines. The quantum numbers of the $X(4140), X(4274), X(4500)$ and $X(4700)$ are according to the LHCb amplitude analysis in Ref. [2]. Note that although the $X(3915)$ is listed with $0^{++}$, it is difficult to understand its properties as the $\chi_{c 0}(2 P)[3,4]$ and it might be just the same state as the $\chi_{c 2}(2 P)$ with $2^{++}[5]$.

\section{Model-independent methods for hadronic molecules}

As mentioned above, the feature of hadronic molecules that they are located close to thresholds makes the investigation of their properties using model-independent EFT approaches possible. Such approaches can be traced back to 1960's, and Weinberg showed that the deutron could not be an elementary particle [6]. The analysis is based on the observation that the probability of finding a physical state in the two-body continuum in an $S$-wave, i.e. the probability of such a state being the corresponding two-body bound state, is related to physical observables. This probability is sometimes called compositeness and denoted by $1-Z$. Its relations to the coupling constant $g_{\mathrm{NR}}$ (here the subscript 'NR' indicates that the nonrelativistic normalization is used), scattering length $a$ and effective range $r_{e}$ are as follows [6-8]:

$$
g_{\mathrm{NR}}^{2} \approx(1-Z) \frac{2 \pi}{\mu^{2}} \sqrt{2 \mu E_{B}}, \quad a \approx-\frac{2(1-Z)}{(2-Z) \sqrt{2 \mu E_{B}}}, \quad r_{e} \approx \frac{Z}{(1-Z) \sqrt{2 \mu E_{B}}} .
$$

These relations receive corrections of order $r \sqrt{2 \mu E_{B}}$ with $r$, typically $\sim 1 / \Lambda_{\text {hadron }}$, the range of forces. Therefore, for an $S$-wave near-threshold state, if the partial decay widths into the modes with a small energy release, which are sensitive to the coupling constants, can be measured precisely, ${ }^{1}$ one can then extract the probability for that state to be made of two hadrons. If the probability is large, then the state is predominantly a hadronic molecule.

\footnotetext{
${ }^{1}$ It would be difficult to measure the scattering parameters experimentally for two heavy hadrons, yet they could be calculated using lattice QCD.
} 
In combination with the heavy quark symmetry to be discussed below, specific EFTs for the nearthreshold $X Y Z$ states as hadronic molecules exist, such as the ones in [9-13]. The above mentioned model-independent relations are built in these EFTs explicitly or implicitly. It is also clear that the coupling constant is bound from above and the upper bound corresponds to the case with $Z=0$. Thus, the larger the hadronic molecular component, the larger the coupling constant, or vice versa. It is then natural to expect that transitions between two hadronic molecules with a small mass difference are more easily to take place than those between states dominated by $Q \bar{Q}$. In Ref. [12], using a nonrelativistic EFT, we calculated the transition $Y(4260) \rightarrow \gamma X(3872)$ assuming the $Y(4260)$ and $X(3872)$ to be $D_{1} \bar{D}[14]$ and $D \bar{D}^{*}$ molecules $[15,16]$, respectively, and predicted a large partial width. The experimental observation of such a decay was soon reported by the BESIII Collaboration [17].

In the limit of the heavy quark mass $m_{Q} \rightarrow \infty$, the heavy quark spin decouples. As a result, we have heavy quark spin symmetry (HQSS). If we decompose the total spin of a hadronic system into two parts: $\vec{J}=\vec{s}_{Q}+\vec{s}_{\ell}$ with $s_{Q}$ the total spin of all the heavy quarks and $s_{\ell}$ the total anglular momentum of the light degrees of freedom, then angular momentum conservation implies that $s_{\ell}$ is a good quantum number as well in the heavy quark limit. We thus have spin multiplets, which are degenerate for $m_{Q} \rightarrow \infty$, for both singly heavy hadrons and heavy quarkonia, such as the $\left(B, B^{*}\right)$ and $\left(\eta_{b}, \Upsilon\right)$, respectively.

Now let us discuss the consequences of HQSS for hadronic molecules with hidden $Q \bar{Q}$. If the hadronic molecule is a loosely bound state of a heavy quarkonium and light hadrons, then because they can only interact through the exchange of at least two gluons, HQSS would lead to a spin multiplet of hadronic molecules [18]. This was used to predict the properties of an $\eta_{c}^{\prime} f_{0}(980)$ bound state with $J^{P C}=0^{-+}[18]$ as the spin partner of the $Y(4660)$ were it a $\psi^{\prime} f_{0}(980)$ bound state [19]. The same analysis in fact can also be applied to hadro-quarkonia as discussed in Ref. [20].

For the hadronic molecules formed by a pair of heavy and anti-heavy mesons, the HQSS structure is more complicated than the above case. As an example, we consider the $S$-wave interaction between a pair of $s_{\ell}^{P}=\frac{1}{2}^{-}$(anti-)heavy mesons. We have a basis of six states of meson pairs:

$$
\begin{aligned}
& 0^{++}: \quad D \bar{D}, \quad D^{*} \bar{D}^{*}, \quad 1^{+-}: \frac{1}{\sqrt{2}}\left(D \bar{D}^{*}+D^{*} \bar{D}\right), \quad D^{*} \bar{D}^{*}, \\
& 1^{++}: \frac{1}{\sqrt{2}}\left(D \bar{D}^{*}-D^{*} \bar{D}\right), \quad 2^{++}: D^{*} \bar{D}^{*}
\end{aligned}
$$

Because of HQSS, the interaction at leading order is independent of the heavy quark spin, and thus can be described by the matrix elements $\left\langle s_{\ell 1}^{\prime}, s_{\ell 2}^{\prime}, s_{L}|\hat{\mathcal{H}}| s_{\ell 1}, s_{\ell 2}, s_{L}\right\rangle$ where $s_{\ell 1, \ell 2}$ and $s_{\ell 1, \ell 2}^{\prime}$ are for the heavy mesons in the initial and final states, respectively, $s_{L}$ is the total angular momentum of the light quarks in the meson-pair system. Thus, for the systems under consideration, we have two independent terms for each isospin $(I=0$ or 1$)$ : $\left\langle\frac{1}{2}, \frac{1}{2}, 0|\hat{\mathcal{H}}| \frac{1}{2}, \frac{1}{2}, 0\right\rangle$ and $\left\langle\frac{1}{2}, \frac{1}{2}, 1|\hat{\mathcal{H}}| \frac{1}{2}, \frac{1}{2}, 1\right\rangle$. This simple observation leads to the conclusion that in the strict heavy quark limit the six pairs in Eq. (2) are grouped in two multiplets with $s_{L}=0$ and 1, respectively. In the limit $m_{c} \rightarrow \infty, D$ and $D^{*}$ are degenerate, it is convenient to use the basis of states $s_{L}^{P C} \otimes s_{c \bar{c}}^{P C}$ with $s_{c \bar{c}}$ refers to the total spin of the $c$ and $\bar{c}$ pair. For the case of $S$-wave interaction, both of $s_{L}^{P C}$ and $s_{c \bar{c}}^{P C}$ can only be $0^{-+}$or $1^{--}$. Therefore, the spin multiplet with $s_{L}=0$ contains two states with quantum numbers:

$$
0_{L}^{-+} \otimes 0_{c \bar{c}}^{-+}=0^{++}, \quad 0_{L}^{-+} \otimes 1_{c \bar{c}}^{--}=1^{+-},
$$

and the spin multiplet for $s_{L}=1$ has the following four states:

$$
1_{L}^{--} \otimes 0_{c \bar{c}}^{-+}=1^{+-}, \quad 1_{L}^{--} \otimes 1_{c \bar{c}}^{--}=0^{++} \oplus 1^{++} \oplus 2^{++}
$$


It becomes clear that if the $1^{++}$state $X(3872)$ is a $D \bar{D}^{*}$ molecule, then it is in the multiplet with $s_{\ell}=1$ [21], and has three spin partners with $J^{P C}=0^{++}, 2^{++}$and $1^{+-}$in the strict heavy quark limit, as pointed out in Refs. [22, 23]. This is also the reason why it was suggested that the $Z_{b}(10610)$ and $Z_{b}(10650)$ might have four more isovector partners $W_{b 0}^{(\prime)}, W_{b 1}$ and $W_{b 2}$ [24-26].

It is worthwhile to notice that the two $1^{+-}$states are in different multiplets with $s_{\ell}=0$ and 1 , respectively, and thus cannot be related to each other via HQSS. However, the isovector $Z_{b}(10610)$ and $Z_{b}(10650)$ are located with similar distances to the $B \bar{B}^{*}$ and $B^{*} \bar{B}^{*}$ thresholds, respectively. Such an approximate degeneracy implies that the isovector interactions in the $s_{\ell}=0$ and $s_{\ell}=1$ sectors are approximately the same, and the off-diagonal transition strength in the isovector channel between the two meson pairs with $J^{P C}=1^{+-}$in Eq. (2) approximately vanishes. ${ }^{2}$ This leads to the "light quark spin symmetry' hypothesis proposed by Voloshin very recently [28]. A deeper understanding for such a phenomenon is still needed. Meanwhile, it is worthwhile to notice the analysis that channel-coupling effects could be neglected based on power counting arguments [29]. Neglecting channel coupling, the charm partners for the $Z_{b}$ states were predicted in Ref. [30], which could correspond to the charged $Z_{c}(3900)$ [31, 32] and $Z_{c}(4020)$ [33] observed by the BESIII and Belle collaborations.

When the physical nondegenerate masses for the heavy mesons are used, one needs the physical particle basis given in Eq. (2), and the two-multiplet pattern gets changed. The effective Lagrangian for the leading order calculation was given in Ref. [9]. It turns out that the contact interactions for the $1^{++}$and $2^{++}$channels are the same. One generally expects a $2^{++} D^{*} \bar{D}^{*}$ bound state assuming the $X(3872)$ to be a $D \bar{D}^{*}$ bound state [22]. Such a $X_{2}$ state should decay dominantly into the $D \bar{D}$ in a $D$-wave, and the width could be up to a few tens of $\mathrm{MeV}[23,34]$. The complexity on the $X_{2}$ due to the coupling of bare quark model $c \bar{c}$ poles was recently examined in Ref. [35].

\section{What can we say about productions and decays of hadronic molecules?}

Nowadays lots of phenomenological studies of hadron spectrum are concentrated on candidates of exotic hadrons, and what the nature of the observed $X Y Z$ states is. Many of such calculations focus on the mass spectrum, and suggest certain assignments to the observations using the methods of quark model, QCD sum rules and so on. However, no assertive statements can be made on the nature of these experimental structures based solely on the mass. On one hand, different models can produce the same mass, in particular if one only study a single state, by adjusting parameters, which is one of the reasons for the uncontrollable inherent uncertainty of such calculations. On the other hand, as discussed in the preceding section, it is the coupling strength that contains important information on the nature. Therefore, for the purpose of understanding the $X Y Z$ structures, it is unavoidable to study carefully their decays and productions which depend on the coupling strengths. In this regard, it is worthwhile to make the following comment: in an EFT, it is necessary to include all the relevant lowenergy degrees of freedom, unless their couplings are very weak, in order to describe the system under consideration properly. In this spirit, no matter what model is used to describe a structure close to the threshold of a strongly coupled channel, the effect of that channel has to be taken into account. This is also the reason for the nowadays well-known pattern that the observed charmoium spectrum higher than open-charm thresholds does not agree with the quark model predictions, as shown in Fig. 1.

One sees the peculiarity of hadronic molecules as the closeness of their masses to the corresponding thresholds allows for a model-independent nonrelativistic EFT treatment. The EFT treatment applies if all the dynamical momenta in the involved processes are small, i.e. for long-distance processes. Yet, this also means that once we go to the processes happening at short distances which

\footnotetext{
${ }^{2} \mathrm{~A}$ fit to the Belle data of the line shapes of the $Z_{b}$ states with HQSS constraints implemented also leads to nearly vanishing channel coupling [27].
} 
contain dynamics inaccessible in the low-energy EFT, model-independence would be lost without further inputs. We thus conclude that hadronic molecules, as extended objects with a size larger than the normal hadrons, can be best studied in long-distance processes with a small energy release, while the short-distance processes are insensitive to the hadronic molecular component of physical states. Examples of processes dominated by the short-distance physics include productions of the $X(3872)$ state in high-energy hadron colliders at high- $p_{T}$, in $B$ decays and the decay of the $X(3872)$ into the $J / \psi \gamma$. Examples of long-distance processes for the $X(3872)$ include the decays of the $X(3872)$ into $D^{0} \bar{D}^{0} \pi^{0}$ and $D^{0} \bar{D}^{0} \gamma$. Measuring the latter processes precisely would be very important in understanding the nature of the $X(3872)$. These decay modes have been discussed by various groups, e.g., Refs. [10, 16, 36-41].

The $X(3872) \rightarrow D^{0} \bar{D}^{0} \pi^{0}$ decay has been already observed by the Belle Collaboration [42, 43], which triggered the virtual state explanation for the $X(3872)$ [44]. If the final state interaction (FSI) between the charmed mesons can be neglected, the predictions can be reliably predicted [10]. However, the strength of the interaction between the charmed meson pairs in the $s_{\ell}=0$ sector in Eq. (3) is not known. It is possible that the interaction is attractive and is strong enough to produce a nearthreshold pole in the $0^{++}$channel to which the $D^{0} \bar{D}^{0}$ couples. In this case, the partial width of $X(3872) \rightarrow D^{0} \bar{D}^{0} \pi^{0}$ will be largely modified by the $D \bar{D}$ FSI as discussed in details in Ref. [41].

\section{Summary}

We have emphasized that hadronic molecules can only be sensibly defined as near-threshold states. This allows them to be treated model-independently using EFT methods. For hadronic molecules with hidden heavy $Q \bar{Q}$, interesting insights can be obtained using HQSS. The hadronic molecular component for a near-threshold state can be directly studied by long-distance decay or production processes instead of the short-distance ones. Therefore, it is important to measure precisely the masses and the long-distance decays for the hadronic molecule candidates such as the $X(3872)$.

I would like to thank M. Albaladejo, C. Hanhart, C. Hidalgo-Duque, Y. Kalashnikova, U. Meißner, A. Nefediev, J. Nieves, A. Ozpineci and M. Pavón Valderrama for the fruitful collaborations and insights on the topics discussed here. This work is supported in part by DFG and NSFC through funds provided to the SinoGerman CRC 110 "Symmetries and the Emergence of Structure in QCD" (NSFC Grant No. 11621131001), by the Thousand Talents Plan for Young Professionals, and by the Chinese Academy of Sciences (Grant No. QYZDBSSW-SYS013).

\section{References}

[1] S. Godfrey and N. Isgur, Phys. Rev. D 32, 189 (1985)

[2] R. Aaij et al. [LHCb Collaboration], arXiv:1606.07898 [hep-ex]

[3] F.-K. Guo and U.-G. Meißner, Phys. Rev. D 86, 091501 (2012)

[4] S. L. Olsen, Phys. Rev. D 91, 057501 (2015)

[5] Z. Y. Zhou, Z. Xiao and H. Q. Zhou, Phys. Rev. Lett. 115, 022001 (2015)

[6] S. Weinberg, Phys. Rev. 137, B672 (1965)

[7] V. Baru, J. Haidenbauer, C. Hanhart, Y. Kalashnikova and A. E. Kudryavtsev, Phys. Lett. B 586, $53(2004)$

[8] T. Hyodo, Int. J. Mod. Phys. A 28, 1330045 (2013)

[9] M. T. AlFiky, F. Gabbiani and A. A. Petrov, Phys. Lett. B 640, 238 (2006)

[10] S. Fleming, M. Kusunoki, T. Mehen and U. van Kolck, Phys. Rev. D 76, 034006 (2007) 
[11] J. Nieves and M. P. Valderrama, Phys. Rev. D 84, 056015 (2011)

[12] F.-K. Guo, C. Hanhart, U.-G. Meißner, Q. Wang and Q. Zhao, Phys. Lett. B 725, 127 (2013)

[13] E. Braaten, Phys. Rev. D 91, 114007 (2015)

[14] Q. Wang, C. Hanhart and Q. Zhao, Phys. Rev. Lett. 111, 132003 (2013)

[15] N. A. Törnqvist, Phys. Lett. B 590, 209 (2004)

[16] M. B. Voloshin, Phys. Lett. B 579, 316 (2004)

[17] M. Ablikim et al. [BESIII Collaboration], Phys. Rev. Lett. 112, 092001 (2014)

[18] F.-K. Guo, C. Hanhart and U.-G. Meißner, Phys. Rev. Lett. 102, 242004 (2009)

[19] F.-K. Guo, C. Hanhart and U.-G. Meißner, Phys. Lett. B 665, 26 (2008)

[20] M. Cleven, F.-K. Guo, C. Hanhart, Q. Wang and Q. Zhao, Phys. Rev. D 92, 014005 (2015)

[21] M. B. Voloshin, Phys. Lett. B 604, 69 (2004)

[22] C. Hidalgo-Duque, J. Nieves, A. Ozpineci and V. Zamiralov, Phys. Lett. B 727, 432 (2013)

[23] V. Baru, E. Epelbaum, A. A. Filin, C. Hanhart, U.-G. Meißner and A. V. Nefediev, arXiv:1605.09649 [hep-ph]

[24] A. E. Bondar, A. Garmash, A. I. Milstein, R. Mizuk and M. B. Voloshin, Phys. Rev. D 84, $054010(2011)$

[25] M. B. Voloshin, Phys. Rev. D 84, 031502 (2011)

[26] T. Mehen and J. W. Powell, Phys. Rev. D 84, 114013 (2011)

[27] F.-K. Guo, C. Hanhart, Y. S. Kalashnikova, P. Matuschek, R. V. Mizuk, A. V. Nefediev, Q. Wang and J.-L. Wynen, Phys. Rev. D 93, 074031 (2016)

[28] M. B. Voloshin, Phys. Rev. D 93, 074011 (2016)

[29] M. P. Valderrama, Phys. Rev. D 85, 114037 (2012)

[30] F.-K. Guo, C. Hidalgo-Duque, J. Nieves and M. P. Valderrama, Phys. Rev. D 88, 054007 (2013)

[31] M. Ablikim et al. [BESIII Collaboration], Phys. Rev. Lett. 110, 252001 (2013)

[32] Z. Q. Liu et al. [Belle Collaboration], Phys. Rev. Lett. 110, 252002 (2013)

[33] M. Ablikim et al. [BESIII Collaboration], Phys. Rev. Lett. 111, 242001 (2013)

[34] M. Albaladejo, F.-K. Guo, C. Hidalgo-Duque, J. Nieves and M. P. Valderrama, Eur. Phys. J. C 75, 547 (2015)

[35] E. Cincioglu, J. Nieves, A. Ozpineci and A. U. Yilmazer, arXiv:1606.03239 [hep-ph].

[36] E. S. Swanson, Phys. Lett. B 588, 189 (2004)

[37] M. B. Voloshin, Int. J. Mod. Phys. A 21, 1239 (2006)

[38] W. H. Liang, R. Molina and E. Oset, Eur. Phys. J. A 44, 479 (2010)

[39] E. Braaten and J. Stapleton, Phys. Rev. D 81, 014019 (2010)

[40] V. Baru, A. A. Filin, C. Hanhart, Y. S. Kalashnikova, A. E. Kudryavtsev and A. V. Nefediev, Phys. Rev. D 84, 074029 (2011)

[41] F.-K. Guo, C. Hidalgo-Duque, J. Nieves, A. Ozpineci and M. P. Valderrama, Eur. Phys. J. C 74, $2885(2014)$

[42] G. Gokhroo et al. [Belle Collaboration], Phys. Rev. Lett. 97, 162002 (2006)

[43] T. Aushev et al. [Belle Collaboration], Phys. Rev. D 81, 031103 (2010)

[44] C. Hanhart, Y. S. Kalashnikova, A. E. Kudryavtsev and A. V. Nefediev, Phys. Rev. D 76, 034007 (2007) 